\title{
PREVALENCE AND ASSOCIATED ERGONOMIC FACTORS OF RADIAL STYLOID TENOSYNOVITIS AMONG ELECTRONIC ASSEMBLY WORKERS IN THAILAND
}

Somthus Pullopdissakul, Chatchai Ekpanyaskul, Sasitorn Teptrakarnporn, Adul Bundhukul, Arunwongse Thepchatri SWU, Bangkok, Thailand

10.1136/oemed-2011-100382.241

Objectives to determine the magnitude, distribution and associated ergonomic factors of radial styloid tenosynovitis among electronic assembly workers in Thailand.

Methods the design was a cross-sectional study. 591 of 853 workers in an electronic and electrical appliance assembly factory in Bangkok, Thailand, participated in the study during October 2010 to January 2011. The self-administered questionnaire consisted of demographic data and ergonomic factors. The clinical examination was done by a physician for radial styloid tenosynovitis diagnosis. The criteria for diagnosis came from the consensus by a group of orthopaedists. The associated ergonomic factors of radial styloid tenosynovitis were analysed by multiple logistic regression.

Results the prevalence of radial styloid tenosynovitis in electronic assembly workers was $13.08 \%$ (95\% CI 10.23 to $15.92 \%)$. They were aged $18-30$ years. $97.46 \%$ were female. After adjustment for age, gender, education and body mass index, the adjusted ORs to develop radial styloid tenosynovitis for the following ergonomic factors were: forceful exertion of wrist 1.78 (95\% CI 1.06 to 2.99 ), awkward posture of wrist 2.37 (95\% CI 1.28 to 4.36 ) and contact stress at wrists 1.75 (95\% CI 1.02 to 3.00). However, there was no association between repetitive motion and radial styloid tenosynovitis.

Conclusions radial styloid tenosynovitis was the most common upper extremities musculoskeletal disease in electronic assembly workers. The ergonomic risk factors for this problem were forceful exertion, awkward posture and contact stress. The relevant parties should provide ergonomic training for these workers. 